\title{
Ion Transport, Selectivity, and Electronic Polarization in Fluoride Channels
}

\author{
Zhi Yue," Zhi Wang, ${ }^{\#}$ and Gregory A. Voth ${ }^{*}$ \\ Department of Chemistry, Chicago Center for Theoretical Chemistry, James Franck Institute, and Institute \\ for Biophysical Dynamics, The University of Chicago, Chicago, Illinois 60637, United States \\ *Correspondence: gavoth@uchicago.edu \\ \#Authors contributed equally to this work.
}

ABSTRACT Fluoride channels (Fluc) export toxic $\mathrm{F}^{-}$from the cytoplasm. Crystallography and mutagenesis have identified several conserved residues crucial for fluoride transport, but the transport mechanism at the molecular level has remained elusive. Herein we have applied constant-pH molecular dynamics and free energy sampling methods to investigate fluoride transfer through a Fluc protein from Escherichia coli. We find that fluoride is facile to transfer in its charged form, i.e., $\mathrm{F}^{-}$, by traversing through a non-bonded network. The extraordinary $\mathrm{F}^{-}$selectivity is gained by the hydrogen-bonding capability of the central binding site and the Coulombic filter at the channel entrance. The $\mathrm{F}^{-}$transfer rate calculated using an electronically polarizable force field is significantly more accurate compared to the experimental value than that calculated using a more standard additive force field, suggesting an essential role for electronic polarization in the $\mathrm{F}^{-}-$Fluc interactions.

SIGNIFICANCE A comprehensive atomistic-level computational study is presented of the mechanism of fluoride transport through fluoride channels. The mechanism of fluoride transfer of the $\mathrm{F}^{-}$anion is established and the microscopic determinants of $\mathrm{F}^{-}$selectivity revealed. The essential nature of electronic polarization during $\mathrm{F}^{-}$transport is also demonstrated through the computational modeling.

\section{INTRODUCTION}

Fluoride is ubiquitous in the biosphere at $20-100 \mu \mathrm{M}$ base levels (1). To alleviate fluoride toxicity $(2,3)$, many organisms utilize transmembrane proteins to reduce cytoplasmic $\mathrm{F}^{-}$accumulation: $\mathrm{F}^{-} / \mathrm{H}^{+}$antiporters $\left(\mathrm{CLC}^{\mathrm{F}}\right.$ ) found in eubacteria (4), and more broadly distributed $\mathrm{F}^{-}$channels (Fluc, also known as CrcB in bacteria or FEX in eukaryotes) (5).

The Fluc family's function (6-14) and structure (10,11,15-19) have been studied extensively since its discovery (20-24). Though expressed as a homodimer, two bacterial Fluc monomers work independently so that inactivating one does not block $\mathrm{F}^{-}$conduction through the other (10). The monomers transfer $\mathrm{F}^{-}$ unidirectionally, as the single-channel conductance is about half of the double-channel value (10). Fluc has a measured turnover rate of $10^{6}-10^{7}$ ions/s $(6,8)$, a rate typical for ion channels. Notably, Fluc is exceptionally selective towards $\mathrm{F}^{-}$over $\mathrm{Cl}^{-}$and small cations $(6,8)$.

Crystal structures from Escherichia coli. (Fluc-Ec2, PDB: 5A43) and Bordetella pertussis (Fluc-Bpe, PDB: 5NKQ) have revealed an antiparallel dimer with two segregated $\mathrm{F}^{-}$pores (Fig. 1A) (16). In the $5 \mathrm{NKQ}$ structure, each monomer harbors two $\mathrm{F}^{-}$ions. One $\mathrm{F}^{-}$is seen in the center (hence denoted as $\mathrm{S}_{\mathrm{cen}}$ ) 
interacting with a conserved F82 (F80 in Fluc-Ec2) from its twin monomer via edgewise anion-aromatic interaction (Fig. 1B). The second $\mathrm{F}^{-}$is situated near the pore exit (hereafter termed as $\mathrm{S}_{\text {ext }}$ ) and stabilized via hydrogen-bond (HBond) with a strongly conserved N43 (N41 in Fluc-Ec2) and anion-aromatic interaction with a conserved F85 (F83 in Fluc-Ec2). The 5A43 structure only has two $\mathrm{S}_{\text {cen }} \mathrm{F}^{-}$ions. The side-chains of the four phenylalanines side-to-face assemble into a symmetrical motif ("Phe-box", Fig. 1C) that has been demonstrated to be essential for fluoride transport $(10,16)$. Beyond $S_{\text {ext }}$, there is another binding site $\mathrm{S}_{\text {out }}$ in some Fluc-Ec2 structures, where $\mathrm{F}^{-}$sits near E86 (E88 in Fluc-Bpe, Fig. 1B). The fourth binding site $S_{\text {int }}$ at the pore entrance is observed in Fluc-Ec2 mutants crystallized with $\mathrm{Br}^{-}$(19). $S_{\text {int }}$ anions interact with twin monomer via HBonds with the side-chain hydroxyls of an invariant S81 (S83 in Fluc-Bpe) and a highly conserved T82 (T84 in Fluc-Bpe), as well as ion-pair (or salt-bridge) with a universally conserved R19 (R23 in Fluc-Bpe) (Fig. 1B). Table S1 of the Supporting Material summarizes the Fluc crystal structures obtained to date. Modestly conserved polar residues on the fourth helix ("polar track", Y104/S108/S112/T116 in Fluc-Bpe, S102/H106/S110/T114 in Fluc-Ec2, Fig. 1D) are expected to further stabilize $\mathrm{F}^{-}$along the pore. In addition, a $\mathrm{Na}^{+}$cation was found deeply buried at the dimer interface and sandwiched by R23s (Fig. 1AB). This important $\mathrm{Na}^{+}(14)$ was thought to stabilize the dimer and add electropositivity to the pores $(5,16)$.

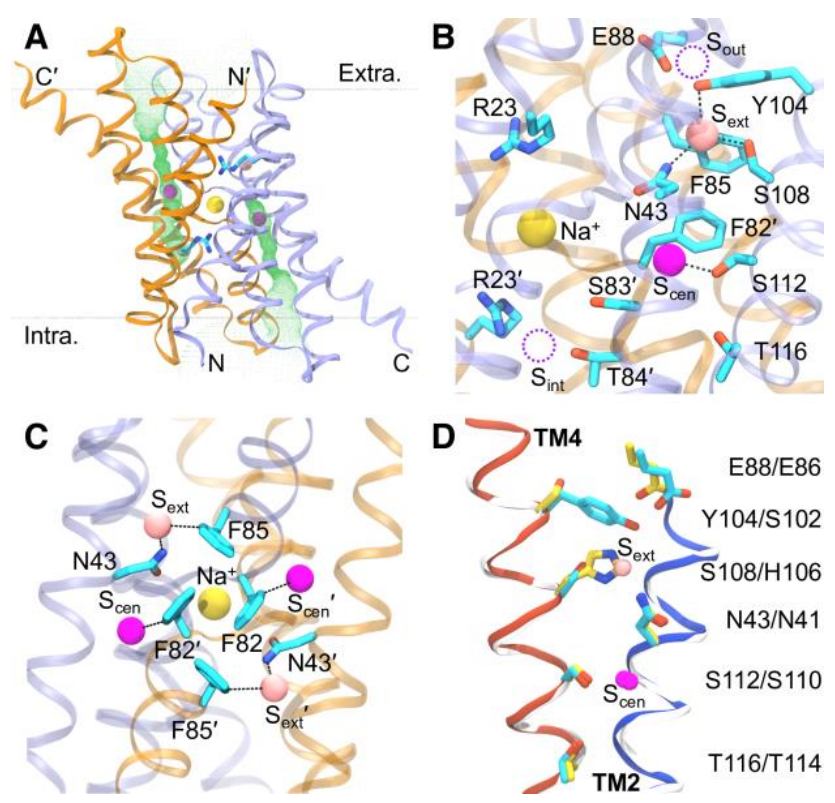

FIGURE 1. Fluc structure and essential residues. (A) Fluc architecture. Fluc-Bpe dimer is shown as ribbon (ice-blue and orange for chains $A$ and $B$, respectively). Ions are represented by spheres $\left(\mathrm{Na}^{+}\right.$: yellow; $\mathrm{S}_{\mathrm{cen}} \mathrm{F}^{-}$: magenta; $\mathrm{S}_{\text {out }} \mathrm{F}^{-}$: pink). R23s are displayed as sticks. Green mesh depicts the pores generated by HOLE (25). Black dots indicate the lipid head groups calculated by the PPM webserver (26). Bilayer orientation and Fluc termini are labeled. Primed labels belong to chain B. (B) $\mathrm{F}^{-}$-binding sites. Essential residues and ions are labeled. Black dashed lines indicate HBonds. Note $S_{\text {int }}$ and $S_{\text {out }}$ (dashed purple circles) were seen in Fluc-Ec2. (C) "Phe-box". Black dashed lines indicate HBonds (with N43s) or edgewise anion-aromatic interactions (with F82s/F85s). (D) "Polar track". Helices 2 and 4 from Fluc-Bpe (blue and red) and Fluc-Ec2 (white) are overlaid. Relevant residues (cyan and yellow for Fluc-Bpe and Fluc-Ec2 carbon atoms, respectively) and $\mathrm{F}^{-}$ions are labeled. Note the $S_{\text {ext }} \mathrm{F}^{-}$is seen in Fluc-Bpe. The presentation styles adopted here are used throughout the paper unless otherwise stated.

Nevertheless, the fluoride transport mechanism remains elusive. Given the HBond donors lining the diffusion pores, it has been assumed that fluoride permeates Flucs in its charged state, i.e., $\mathrm{F}^{-}$(16). 
However, as the transfer energetics has not been established, it is unclear if and how Flucs could compensate for the high desolvation penalty of $\mathrm{F}^{-}(111 \mathrm{kcal} / \mathrm{mol}$, Ref. (27)) upon its entry. A eukaryotic Fluc from Camellia sinensis has been shown to couple $\mathrm{H}^{+}$gradient with fluoride efflux (28), but the same coupling has not yet been reported in its bacterial counterparts. The fact that the identity of the "polar track" residues varies among Flucs adds to the mystery. Fluc-Ec2 comprises an ionizable "track" residue H106 (Fig. 1C). Replacement of H106, even by HBond substitutes like H106S (Fluc-Bpe equivalence), makes Fluc-Ec2 completely inactive (11), suggesting that H106 is more than an HBond donor. Since H106 lies near the pore end and is (at least partially) solvated, it is likely to be protonated under physiological pHs to coordinate with F83 via cation- $\pi$ interactions (Fig. 1BD). Titration of H106 or $\mathrm{F}^{-}$might be necessary for $\mathrm{F}^{-}$bypass to avoid electrostatic trapping. The absence of an ionizable counterpart in other Flucs would suggest either no titration of $\mathrm{H} 106 / \mathrm{F}^{-}$in Fluc-Ec2 or wide variance in the transport mechanism across the family. Other "track" residues in Fluc-Ec2 (S102/S110/T114) are insensitive to mutagenesis (11). The lack of necessity of HBond donors at these locations with conserved polarity thus adds more complexity. These mechanical puzzles require a comprehensive assessment of the charge state of fluoride inside Fluc and the accompanying transport energetics.

Electronic polarization may be essential during ion permeation through some inhomogeneous membrane systems $(29,30)$. This is one major challenge for computational studies using standard nonpolarizable additive force fields (FFs), which approximate the response to different dielectric media in a mean-field fashion (31,32). Additive FFs have, for example, overestimated the transfer barrier of cations $\left(\mathrm{Li}^{+} / \mathrm{Na}^{+} / \mathrm{K}^{+}\right)$ through gramicidin A (gA) channel by $5-10 \mathrm{kcal} / \mathrm{mol}$, and estimated conductance that differed by several orders of magnitude from experimental values (33-36). Zhang et al. (37) noticed that the water wire in gA was more structured with the additive FF description. Ngo et al. (38) further confirmed this FF-dependent water structure. They also revealed the balance between water-water and water-protein interactions impacted the water dynamics inside the channel and changed the energetics of ion permeation. Similar observations have also been reported for potassium (KcsA) and voltage-gated sodium (Nav) channels. Jing et al. (39) discovered polarization played a crucial role in the thermodynamic stabilities of different $\mathrm{K}^{+}$binding configurations. Sun et al. (40) found the free energy barrier of $\mathrm{Na}^{+}$transfer was overestimated by $\sim 2 \mathrm{kcal} / \mathrm{mol}$ using additive FF due to a failure to describe the electrostatics between the charged gating residues and aromatic residues with large polarizability. The anion case has, however, received much less attention. Vergara-Jaque et al. (41) examined the free energy of $\mathrm{I}^{-}$transfer through a sodium-iodide symporter (NIS) and noticed an additive FF missed a global minimum around the putative $\mathrm{I}^{-}$binding site. However, their work studied a fragment of NIS and did not inspect the origin of the FF-dependent energetics. Fluc, a small channel, should thus be an ideal model for assessing the electronic polarization effect during anion permeation.

In this paper, we have investigated the fluoride transport mechanism in Fluc-Ec2 via a combination of in silico approaches. We first applied the continuous constant-pH molecular dynamics (CpHMD) technique $(42,43)$ to examine the charge state of fluoride inside Fluc. In CpHMD, a titration coordinate, $\lambda$, is associated with each ionizable site and propagates simultaneously with the spatial coordinates in the MD. The charge states (or $\lambda$ values) are updated on-the-fly during the simulation and closely coupled to the conformational dynamics. With this approach, we found that $\mathrm{F}^{-}$is stable inside Fluc using the membrane-enabled hybrid-solvent CpHMD $(44,45)$, a formulation that provides high-accuracy $\mathrm{p} K_{\mathrm{a}}$ prediction (46). We then evaluated the free energy of $\mathrm{F}^{-}$transport through Fluc with the replica-exchange umbrella sampling (REUS) method $(47,48)$. We discovered that $\mathrm{F}^{-}$is relayed inside a network of HBonds, ion-pairs, and anion- $\pi$ pairs. The dehydration penalty of anhydrous $S_{\text {cen }} \mathrm{F}^{-}$was mainly offset by the HBonds, suggesting $\mathrm{S}_{\text {cen }}$ is the selectivity filter discriminating $\mathrm{F}^{-}$over $\mathrm{Cl}^{-}$. The selectivity over cations is likely conferred by the "Coulombic plug” R19. Moreover, proper treatment of ion- $\pi$ interactions requires 
explicit treatment of the ion-induced polarization of the $\pi$-electron cloud, which is not well represented in non-polarizable additive FFs (49-51). Thus, we employed both the CHARMM additive CHARMM36m (52-54) and Drude polarizable (55-61) FFs to draw comparisons. The results showed that the additive FF significantly overestimates the ion-protein interactions, leading to an estimated $\mathrm{F}^{-}$flux about six orders of magnitude slower than experimental measurements. Predictions with the polarizable FF, on the other hand, agree well with the experiments, which underlines the importance of electronic polarization during $\mathrm{F}^{-}$transfer in this channel and may point to the utility of polarizable models in studying anion permeation through membrane proteins in general.

\section{METHODS}

The study was conducted following a protocol previously established $(62,63)$ with necessary modifications. For simulations using additive FF, protein, lipids, and waters were represented by CHARMM36m (53,54), CHARMM36 (52), CHARMM-modified (64) TIP3P (65) models, respectively. The parameters for $\mathrm{F}^{-}$and hydrofluoric acid (HF) were reported by Senn et al. (66) and Laage et al. (67), respectively. For CpHMD simulations, the protein was modeled by CHARMM22/CMAP $(68,69)$. In the simulations using polarizable FF, the CHARMM Drude-2013 models (55-60) with cation- $\pi$ and anionring corrections (61) were used. The initial conformation of Fluc-Ec2 was retrieved from the crystal structure (PDB: 5A43) (16) and embedded in a 1-palmitoyl-2-oleoyl-sn-glycero-3-phosphoethanolamine (POPE) lipid bilayer. The system was built using CHARMM-GUI Membrane Builder (70-74) and equilibrated using GROMACS (75) (version 2019.3) for $100 \mathrm{~ns}$. The last snapshot from the equilibration was then converted to CpHMD-compatible format using CHARMM (76) (version c42b2). The membraneenabled hybrid-solvent CpHMD $(44,45)$ with the $\mathrm{pH}$-based replica-exchange enhanced-sampling protocol ( $\mathrm{pH}-\mathrm{REX})(44)$ ran for $20 \mathrm{~ns}$ per replica using CHARMM to calculate the $\mathrm{p} K_{\mathrm{a}} \mathrm{S}$ of fluoride and ionizable residues inside Fluc-Ec2. Twenty-one replicates of the CpHMD system were also generated with the fluoride restrained at various locations along the membrane normal (Z-axis) and equilibrated at $\mathrm{pH} 7 \mathrm{for}$ 50 ns. The equilibrated CpHMD replicates were converted to fixed-protonation-state style using CHARMM for additive and polarizable REUS (48) simulations. The potentials of mean force (PMFs) of $\mathrm{F}^{-}$transfer through Fluc-Ec2 were derived for both sets of systems using NAMD (77). Details of simulations and analysis protocols are described in the Supporting Material.

\section{RESULTS AND DISCUSSION}

\section{Fluoride is charged inside Fluc-Ec2}

We first investigated the charge state of fluoride, i.e., $\mathrm{HF}$ or $\mathrm{F}^{-}$, inside Fluc-Ec2 using the membraneenabled hybrid-solvent CpHMD combined with pH-REX (44,45). All Asp, Glu, and His residues were allowed to ionize in the simulation. $\mathrm{Na}^{+}$-chelating $\mathrm{R} 19 \mathrm{~s}$ were also set to be ionizable to evaluate if they stay charged. The $\mathrm{pH}-\mathrm{REX}$ CpHMD simulation was 20 ns per replica. Calculated $\mathrm{p} K_{\mathrm{a}}$ converged within 10 ns (Fig. S1). Final $\mathrm{p} K_{\mathrm{a}}$ are listed in Table 1. $\mathrm{p} K_{\mathrm{a}}$ for H60s, D62s, and E86s are shifted from their corresponding model compound values $(6.5,3.7,4.3)(78)$ by less than $1 \mathrm{pH}$ units. The "track" residue H106s have significantly upshifted $\mathrm{p} K_{\mathrm{a}} \mathrm{s}$ (8.6 and 9.6 for chain $\mathrm{A}$ and $\mathrm{B}$, respectively), indicating the doubly protonated form is stable around physiological $\mathrm{pH}$. Fluorides and the $\mathrm{Na}^{+}$-sandwiching R19s stay deprotonated and protonated, respectively, within the $\mathrm{pH}$ range studied (3.5-10.5). The $\mathrm{p} K_{\mathrm{as}}$ suggest that at $\mathrm{pH} 7$, where the Fluc-Ec2 $\mathrm{F}^{-}$efflux was measured $(6,10,11)$, fluoride and H106s should stay charged. 
During the simulation, $\mathrm{F}^{-}$ions remained around the $\mathrm{S}_{\mathrm{cen}}$ sites and no $\mathrm{F}^{-}$release was observed, possibly due to limited sampling and overstabilized ion-protein interactions under the additive FF representation (see discussion below).

TABLE 1 Calculated $\mathrm{p} K_{\mathrm{a}} \mathrm{s}$ for ionizable residues/ligands in Fluc-Ec2

\begin{tabular}{|c|c|c|}
\hline \multirow{2}{*}{ Residue } & \multicolumn{2}{|c|}{$\mathrm{p} K_{\mathrm{a}}$} \\
\hline & Chain A & Chain B \\
\hline R19 & \multicolumn{2}{|c|}{ Protonated } \\
\hline H60 & 7.3 & 7.4 \\
\hline D62 & 3.4 & 3.7 \\
\hline E86 & 3.9 & 3.8 \\
\hline H106 & 8.6 & 9.6 \\
\hline HF & \multicolumn{2}{|c|}{ Deprotonated } \\
\hline
\end{tabular}

\section{$F^{-}$transport through Fluc-Ec2 is feasible}

Based on the charge states determined by the pH-REX CpHMD, we then employed the REUS technique $(47,48)$ to evaluate the free energy of $\mathrm{F}^{-}$transport through Fluc-Ec2 using either the C36m $(52-54)$ or Drude (55-61) FFs. Since bacterial Fluc monomers function independently (10), we only modeled $\mathrm{F}^{-}$ transfer through monomer A while restraining the $\mathrm{F}^{-}$in monomer $\mathrm{B}$ around its crystallographic location. Fig. 2A displays the resulting transport PMFs as a function of the distance from the Fluc center $(\Delta \mathrm{ZCOM})$, which delineates the change in free energy as $\mathrm{F}^{-}$travels between internal and external aqueous bulks via Fluc. Both FFs give PMFs (black for C36m, red for Drude) that agree on their overall trend: a rugged free energy landscape with a global minimum close to the $S_{\text {cen }}$ binding site $(\triangle Z C O M$ of $-4 \AA$ ). However, the absolute values of the PMFs differ significantly so that $\mathrm{F}^{-}$is much more stable under the $\mathrm{C} 36 \mathrm{~m}$ representation. In $\mathrm{C} 36 \mathrm{~m}, \mathrm{~F}^{-}$needs to cross a free energy barrier of $17.7 \pm 0.8 \mathrm{kcal} / \mathrm{mol}$, but in Drude, the barrier is $\mathrm{ca}$. $60 \%$ smaller $(7.4 \pm 0.3 \mathrm{kcal} / \mathrm{mol})$. Moreover, the $S_{\text {cen }}$ minimum is only $0.6 \pm 0.5 \mathrm{kcal} / \mathrm{mol}$ more stable than the $S_{\text {ext }}$ one $(\triangle \mathrm{ZCOM}$ of $4 \AA$ ) in the polarizable Drude FF. This suggests that, while slightly favored at $\mathrm{S}_{\mathrm{cen}}, \mathrm{F}^{-}$is also likely to be found at $\mathrm{S}_{\text {ext }}$, consistent with crystal structures $5 \mathrm{~A} 43$ (16), 5NKQ (16), 6BQO (18), 6BX4 (17), and 6BX5 (17) (check Table S1 for details). 

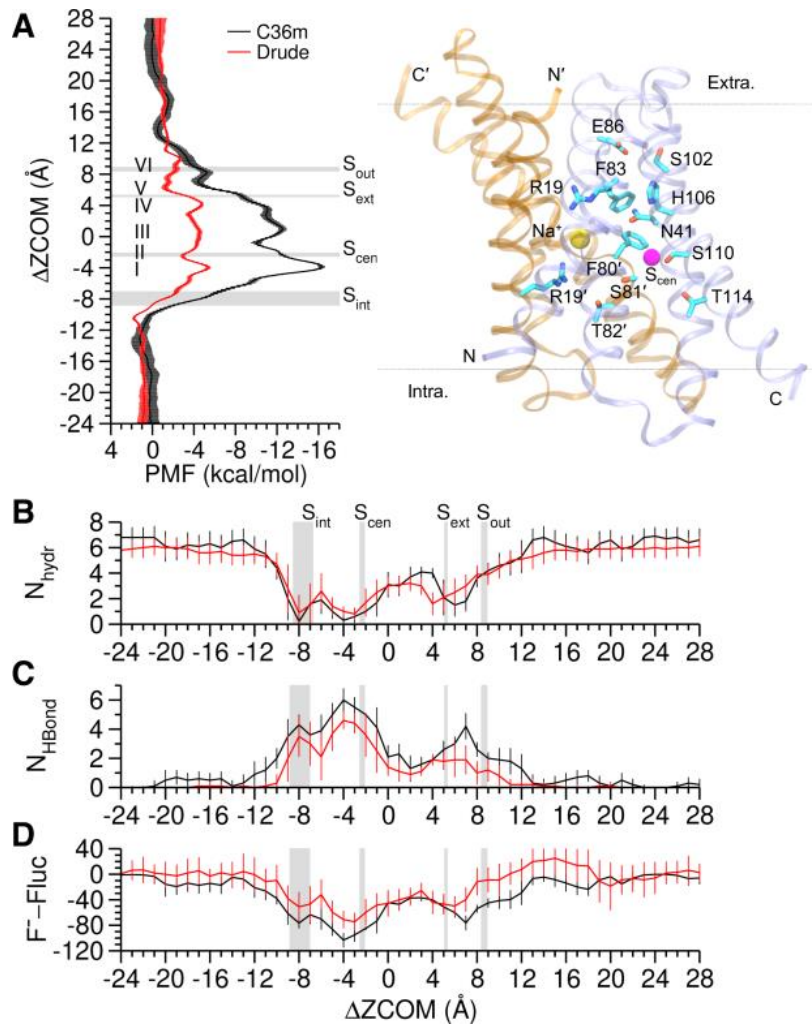

FIGURE 2. $\mathrm{F}^{-}$transport thermodynamics. (A) Potential of mean force (PMF) as a function of $\Delta \mathrm{ZCOM}$, which is defined as the center-of-mass (COM) distance between $\mathrm{F}^{-}$and the Fluc $\mathrm{C} \alpha$-atoms projected on the bilayer normal (Z-axis). PMFs from the additive C36m and polarizable Drude FFs were set to zero in aqueous bulk. Error bars are the standard deviations calculated from block analysis. Grey boxes indicate the crystallographic locations of $\mathrm{S}_{\text {int }}, \mathrm{S}_{\mathrm{cen}}, \mathrm{S}_{\mathrm{ext}}$, and $\mathrm{S}_{\text {out }} \mathrm{F}^{-}$(centroid and halved box width respectively represent the mean and standard deviation calculated from all available PDB structures). Latin alphabet I-VI indicates the representative states illustrated in Fig. 3. The Fluc-Ec2 structure, with essential structural features labeled, is aligned with the PMFs. (B) Hydration number of $\mathrm{F}^{-}$, defined as the number of water oxygen atoms within 3.5 $\AA$ of $\mathrm{F}^{-}$. (C) Number of HBonds between $\mathrm{F}^{-}$and Fluc. HBond is considered to be present if the donor-H distance is $\leq 2.4 \AA$. (D) Interaction energy ( $\mathrm{kcal} / \mathrm{mol})$ of $\mathrm{F}^{-}$with Fluc. The means and fluctuations (shown as error bars) were calculated every $1 \AA$ A along $\triangle \mathrm{ZCOM}$.

To better understand the permeation energetics, we quantified the hydration number $\left(\mathrm{N}_{\text {hydr }}\right)$ and the number of HBonds (NHBond) of $\mathrm{F}^{-}$as a function of $\triangle \mathrm{ZCOM}$. As displayed in Fig. 2B, $\mathrm{F}^{-}$is dehydrated inside Fluc. Yet, the desolvation penalty is compensated by HBonds with Fluc (Fig. 2C), as the $\mathrm{N}_{\text {hydr }}$ and $\mathrm{N}_{\text {HBond }}$ profiles are complementary, regardless of FFs. For instance, the anhydrous $\mathrm{S}_{\mathrm{cen}} \mathrm{F}^{-}$is stabilized by 6 HBonds. The $\mathrm{S}_{\text {ext }} \mathrm{F}^{-}$is solvated by at least 2 water molecules and fewer HBonded. Since NHBond excludes water, our results indicate $\mathrm{F}^{-}$is constantly coordinated with Fluc and water molecules via $\sim 6$ HBonds during the transport, which approximately equals the $\mathrm{N}_{\text {hydr }}$ in aqueous bulk (Fig. 2B). Following the protocol by Lin et al. (79), we calculated the interaction energy (Einter) between $\mathrm{F}^{-}$and Fluc (including the central $\mathrm{Na}^{+}$and the other $\mathrm{F}^{-}$). Consistent with the PMFs, both FFs predict overall favorable Einter of $\mathrm{F}^{-}$ with Fluc, but $\mathrm{E}_{\text {inter }}$ is stronger in $\mathrm{C} 36 \mathrm{~m}$ (Fig. 2D). Residue breakdown of Einter indicates that the C36m overstabilization mainly originates from the interaction with R19' around $S_{\text {int }}$ and $S_{\text {cen, and with R19 }}$ around Sout, which are overestimated by $15-20 \mathrm{kcal} / \mathrm{mol}$ (Fig. S2). Moreover, C36m predicts Einter constantly favorable throughout Fluc, but Drude predicts unfavorable $E_{\text {inter }}$ around $S_{\text {out }}$ and beyond $\left(\triangle \mathrm{ZCOM}>8 \AA\right.$, Fig. 2D), where the negatively charged E86 resides (Fig. 2A, Fig. S3A). Because $\mathrm{F}^{-}$is 
more likely to form contact with E86 in Drude than in C36m (Fig. S3B). Decomposition of Einter indicates unfavorable self-polarization energy ( $E_{\text {self }}$ ), which quantifies the contribution of electronic polarization to

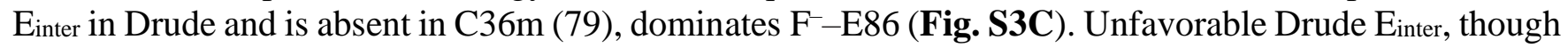
smaller, is also seen with the "Phe-box" (Fig. S2) due to unfavorable Eself (Fig. S4). By contrast, the interactions are marginal in C36m (Fig. S2). The only exception is F80", which strongly interacts with the $\mathrm{S}_{\text {cen }} \mathrm{F}^{-}$via HBond (Fig. 3B).

We next examined the permeation kinetics. The local diffusion constant $D$ was calculated to be $(2.1 \pm$ $0.1) \times 10^{-5}$ and $(1.4 \pm 0.2) \times 10^{-5} \mathrm{~cm}^{2} / \mathrm{s}$ respectively from the $\mathrm{C} 36 \mathrm{~m}$ and Drude REUS simulations (Table 2) using the Woolf-Roux equation (80). The experimental $D$ is $1.475 \times 10^{-5} \mathrm{~cm}^{2} / \mathrm{s}$ in infinitely dilute aqueous solution (81), and $(1.4 \pm 0.1) \times 10^{-5} \mathrm{~cm}^{2} / \mathrm{s}$ considering finite concentration (82). Our results therefore indicate that the Drude FF predicts a correct $D$ whereas $\mathrm{C} 36 \mathrm{~m}$ overestimates $D$ by $50 \%$, which is consistent with the study of ion conductivity by Prajapati et al. (83). The permeation rate constant $k$ was calculated from the PMF and $D$ (Eqs. S2-S3) based on the mean first passage time model (84). The resulting $k$ is $(4 \pm 1) \times 10^{6}$ and $(0.29 \pm 0.26)$ ions/s for Drude and C36m, respectively (Table 2). The Drude estimate agrees with the experimental turnover rate of $10^{6}-10^{7}$ ions/s $(6,8)$. But the C36m estimate is six orders of magnitude slower, far below the typical rate for ion channels. These results further support the viability of $\mathrm{F}^{-}$transport through Fluc and point to nonnegligible electronic polarization during permeation.

TABLE $2 \mathrm{~F}^{-}$bulk diffusion constant $D$ and transport rate constant $k$

\begin{tabular}{ccc}
\hline & $\boldsymbol{D}\left(\mathbf{1 0}^{-\mathbf{5}} \mathbf{c m}^{\mathbf{2}} / \mathbf{s}\right)$ & $\boldsymbol{k}(\mathbf{i o n s} / \mathbf{s})$ \\
\hline Drude & $1.4 \pm 0.2$ & $(4 \pm 1) \times 10^{6}$ \\
C36m & $2.1 \pm 0.1$ & $0.29 \pm 0.26$ \\
Experiment & $1.4 \pm 0.1$ & $10^{6}-10^{7}$ \\
\hline
\end{tabular}

\section{$\mathrm{F}^{-}$relays within a network of non-bonded interactions}

Finally, we investigated the atomistic permeation mechanism based on the Drude simulations. We first computed the occupancies of HBonds and ion-pairs between $\mathrm{F}^{-}$and Fluc polar residues (Fig. 3B) as well as anion- $\pi$ pairs with the "Phe-box" (Fig. 3C) along the pore (i.e., $\Delta$ ZCOM). We then compared these interaction profiles with the transport PMF (Fig. 2A) to extract the most representative patterns (Fig. 3A) for metastable and transition states I-VI (Fig. 2A). 

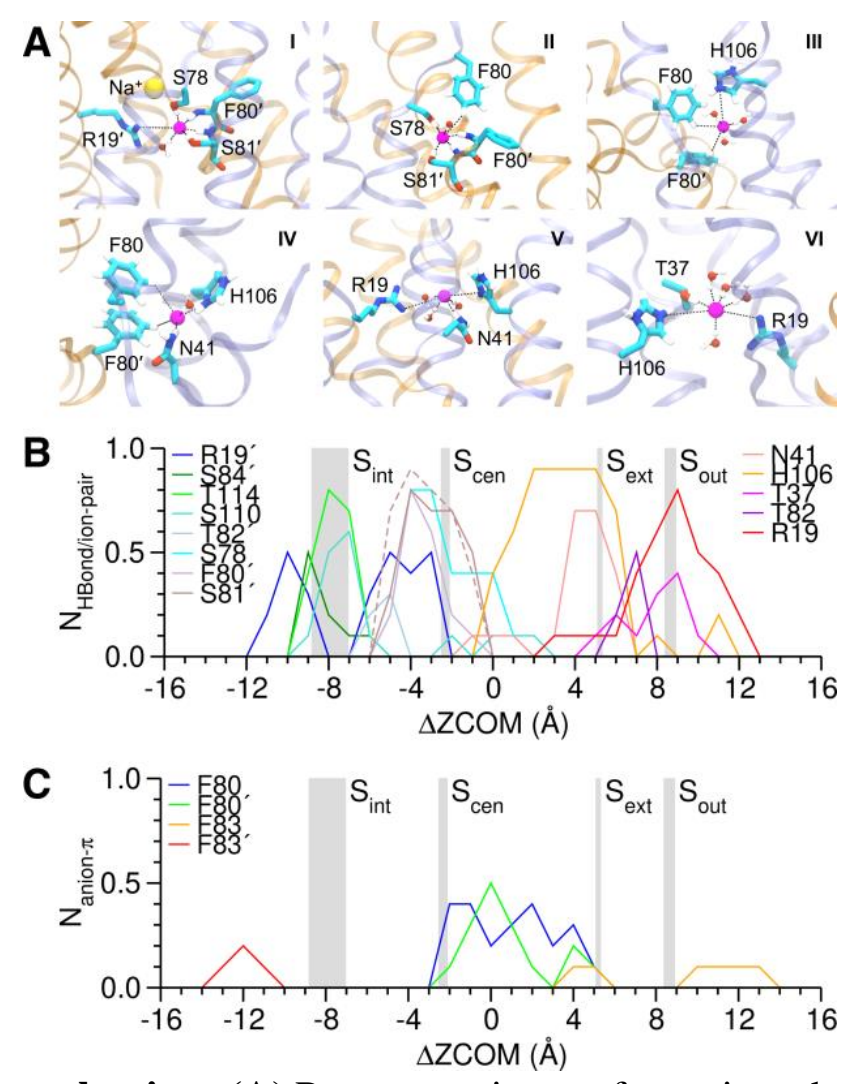

FIGURE 3. $\mathbf{F}^{-}$transport mechanism. (A) Representative conformations during the transport. Latin labels are consistent with Fig. 2A. (B) Occupancies of HBonds/ion-pairs between $\mathrm{F}^{-}$and Fluc. HBond is considered to form if the donor-acceptor distance is $\leq 3.5 \AA$ and the donor-H-acceptor angle is $\geq 150^{\circ}$. Ion-pair is considered to form if the $\mathrm{N}-\mathrm{F}^{-}$minimal distance is $\leq 4.0 \AA$. Note S81' HBonds with $\mathrm{F}^{-}$via backbone (solid line) and side-chain (dashed line). (C) Occupancies of anion- $\pi$ pairs between $\mathrm{F}^{-}$and the "Phe-box". Anion- $\pi$ pair is present if $\mathrm{F}^{-}$is within $4.5 \AA$ of any ring carbon atoms and subtends an angle of $\leq 35^{\circ}$ with the ring plane. For clarity, error bars (fluctuations) are not shown. All data were extracted from the Drude REUS simulations.

In the transport direction (Fig. 2A), $\mathrm{F}^{-}$enters Fluc and binds to $\mathrm{S}_{\text {int }}$ with a sharp decrease in $\mathrm{N}_{\text {hydr }}$ ( $\triangle$ ZCOM from -11 to -5 A , Fig. 2B), but the interactions with R19', S84', T114, and S110 (Fig. 3B) stabilize the desolvated $\mathrm{F}^{-}$. Note $\mathrm{S}_{\text {int }}$ is not a minimum in the transport PMF (Fig. 2A), which explains why $S_{\text {int }}$ was not captured by the structures crystallized with $\mathrm{F}^{-}$. The $\mathrm{F}^{-}$anion translocates from $\mathrm{S}_{\text {int }}$ to $\mathrm{S}_{\mathrm{cen}}$

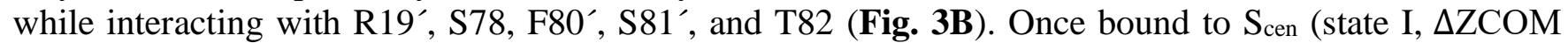
from -5 to $-3 \AA$ ), $\mathrm{F}^{-}$predominantly forms 5 HBonds with S78, F80', S81' (backbone and side-chain), and a water molecule. Meanwhile, $\mathrm{F}^{-}$ion-pairs with R19' (Fig. 3AB). As a result, despite the lower solvation (Fig. 2B), $\mathrm{F}^{-}$is stabilized by the most abundant HBonds and ion-pairs in state I (Fig. 2C), leading to the global minimum in the PMF (Fig. 2A). Note that the $\mathrm{F}^{-}-\mathrm{Na}^{+}$pair is not close enough to be considered as an ion-pair. State II ( $\triangle \mathrm{ZCOM}$ from -3 to $-1 \AA$ ) is the first transition state. Compared to state I, the ionpair with R19' is lost in state II, while the 5 HBonds retain (Fig. 3AB). F $^{-}$moves to the proximity of F80 to form an anion- $\pi$ pair (Fig. 3AC), consistent with the crystal $S_{\text {cen }}$ binding pattern. The anion- $\pi$ pair is not strong enough to balance the ion-pair loss, which explains why state II is less stable. The HBond with S110 (S112 in Fluc-Bpe, Fig. 1B) was also observed in our simulations but with marginal occupancy (Fig. 3B). In state III ( $\triangle \mathrm{ZCOM}$ from -1 to $3 \AA$ ), $\mathrm{F}^{-}$becomes the most solvated (Fig. 2 B) but the least HBonded 
(Fig. 2C). With all the HBonds in states I and II absent, $\mathrm{F}^{-}$begins ion-pairing with H106 (Fig. 3AB). Additional stabilization is provided by anion- $\pi$ pairs with F80 and F80' (Fig. 3AC) and occasionally by HBond with N41 (Fig. 3B), making state III a local minimum in the PMF (Fig. 2A). State IV $(\Delta$ ZCOM from 3 to $5 \AA$ ) is another local minimum in the PMF and corresponds to Sext (Fig. 2A). State IV differs from state III by less solvation (Fig. 2B) and stronger HBond with N41 (Fig. 3AB). The move from state IV to state $\mathrm{V}$ ( $\triangle \mathrm{ZCOM}$ from 5 to $7 \AA$ ), the second transition state, is accompanied by gradual loss of the interactions with F80s and N41 (Fig. 3BC) and by increasing hydration (Fig. 2B). Starting from state V, $\mathrm{F}^{-}$ion-pairs with R19 (Fig. 3AB). State VI ( $\triangle$ ZCOM from 7 to $11 \AA$ ) matches $S_{\text {out }}$ and is the last local minimum in the PMF (Fig. 2A), where the solvated $\mathrm{F}^{-}$is coordinated mainly with R19 and T37 (Fig. 3AB) and infrequently with F83 (Fig. 3C).

Our results show that $\mathrm{F}^{-}$permeates by relaying inside a non-bonded network. The identified interaction patterns highlight the indispensability of conserved or immutable residues. Most of the Fluc interior is an HBond “desert" except around $\mathrm{S}_{\text {cen }}$ (Fig. 2C). Thus, extra stabilization is required to offset the desolvation penalty and help $\mathrm{F}^{-}$transport. First, beyond $\mathrm{S}_{\mathrm{cen}}(\triangle \mathrm{ZCOM}>-1 \AA), \mathrm{F}^{-}$primarily HBonds with $\mathrm{N} 41$ and ion-pairs with H106 ( $\triangle$ ZCOM from 2 to $6 \AA$, Fig. 3B). Within the "gap", the anion- $\pi$ pairs with F80s govern (Fig. 3C), consistent with the observation that F80 mutants are nonfunctional $(10,16)$. Next, N41 and H106 stabilize and guide $\mathrm{F}^{-}$to $\mathrm{S}_{\text {ext }}$ (Fig. 3B). Our CpHMD simulation revealed that the $\mathrm{F}^{-}-\mathrm{H} 106$ ionpair facilitates the transport (Fig. S5). This helps to explain the puzzling finding that H106 is irreplaceable (11). Then, the Drude PMF predicts $S_{c e n}$ as a transition state (Fig. 2A), which appears to be a discrepancy as the crystal binding site should be a minimum in PMF. However, most Fluc structures were crystallized around pH 6 and below (Table S1), whereas our simulations modeled pH 7. Given the stronger $\mathrm{F}^{-}-\mathrm{H} 106$ ion-pair at acidic pHs, it is reasonable the crystal $\mathrm{S}_{\mathrm{cen}}$ site upshifts relative to our PMF global minimum (Fig. S5). Lastly, two R19s are found near the two pore openings (Fig. 1A). They likely help retrieve $\mathrm{F}^{-}$ from internal/external bulk regions and provide initial stabilization upon pore entry (Fig. 3B). This scenario also holds for F83s via anion- $\pi$ pairs, although the interactions are weaker (Fig. 3C).

\section{CONCLUDING DISCUSSION}

We have studied the mechanism of fluoride transport through Fluc-Ec2 in silico. CpHMD simulation found a stable $\mathrm{F}^{-}$(Table 1) at the most dehydrated $\mathrm{S}_{\text {cen }}$ site (Fig. 2B). The transport energetics at pH 7 were then established by the REUS free energy calculations. The transport PMF from the electronically polarizable Drude simulations revealed a global minimum around $S_{\text {cen, a slightly less stable local }}$ minimum at $S_{\text {ext, }}$ and a third local minimum at $S_{\text {out }}$ (Fig. 2A), which are consistent with the crystal structures (Table S1). $\mathrm{F}^{-}$is dehydrated during the transport (Fig. 2B), but the desolvation penalty is compensated by HBonds within Fluc (Fig. 2C) so that $\mathrm{F}^{-}$stays as coordinated inside the channel as it is in aqueous bulk. The transport rate estimated from the Drude simulations agrees well with the experimental turnover rate measured at $\mathrm{pH} 7(6,8)$, further supporting that Fluc transfers $\mathrm{F}^{-}$anion.

$\mathrm{F}^{-}$has been proposed to be stabilized by non-bonded interactions with the pore-facing residues during the transfer (16). Our study supports this idea by showing that $\mathrm{F}^{-}$relays inside a non-bonded network and also explains residue conservation/indispensability. R19 acts as an "antenna" for fetching $\mathrm{F}^{-}$from aqueous bulks and, more importantly, stabilizes $\mathrm{F}^{-}$to $\mathrm{S}_{\mathrm{cen}}$ or $\mathrm{S}_{\mathrm{ext}}$ (Fig. 3AB). Though not yet reported in bacterial Flucs, mutating its equivalent in yeast $S$. cerevisiae (FEXsc) significantly reduces $\mathrm{F}^{-}$efflux (8). N41 has further been shown in both bacteria (16) and eukaryotes (8) to be an immutable HBond donor, with sidechain rotation thought to be coupled with $\mathrm{F}^{-}$motion (16). We observed that the HBond with N41 guided the $\mathrm{S}_{\text {cen }} \mathrm{F}^{-}$to $\mathrm{S}_{\text {ext }}$ (Fig. 3AB), supporting the "rotameric switch" conjecture. Polar residues S78, S81, T82, and S84 (T80, S83, T84, and S86 in Fluc-Bpe) were also identified as HBond donors in our simulations 
(Fig. 3B). The residues $\mathrm{S} 78$ and $\mathrm{S} 81$ form strong HBonds with the $\mathrm{S}_{\mathrm{cen}} \mathrm{F}^{-}$. The $\mathrm{T} 82$ helps $\mathrm{F}^{-}$travel from

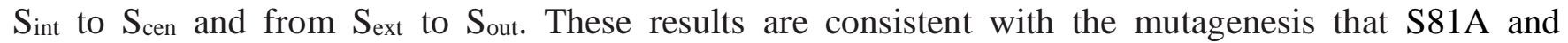
$\mathrm{S} 81 \mathrm{~A} / \mathrm{T} 82 \mathrm{~A}$ respectively cause $\sim 50 \%$ and 100 -fold conductance loss (19). It is unclear, however, why $\mathrm{F}^{-}$ efflux was completely abolished in S81T (19). We speculate that $-\mathrm{CH}_{3}$ is so bulky at $\mathrm{S}_{\text {cen }}$ that it eliminates more HBonds than the one with the S81 side-chain (e.g., the water HBond, Fig. 3A), making $\mathrm{F}^{-}$highly

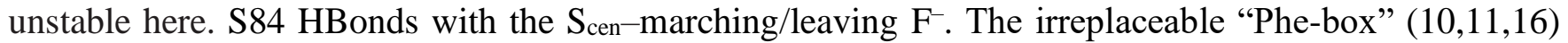
has been hypothesized to form edgewise anion- $\pi$ pairs with $\mathrm{F}^{-}(16)$, which was confirmed by our simulations (Fig. 3C). Notably, we found the anion- $\pi$ pairs with F80s were prominent during the transition between $S_{\text {cen }}$ and $S_{\text {ext. }}$ F80 also HBonds with the $S_{\text {cen }} F^{-}$(Fig. 3B). The anion- $\pi$ pairs with F83s seen near the pore ends are considerably weaker (Fig. 3C). The variation in their pairing abilities is consistent with the observation that Fluc is resistant to F80M rather than F83M (11), because electropositive Met is an anion- $\pi$ substitute for Phe. However, the irreplaceability of F83 remains mysterious.

The "polar track" (Fig. 1D) was proposed to HBond $\mathrm{F}^{-}(16)$, but this argument was challenged by a follow-up mutagenesis study disclosing several paradoxes (11). First, mutations S102A and S110A do not impair Fluc function. No HBond with S102 was seen in our simulations. F- HBonds with S84', T114, and S110 in the same region (Fig. 3B), so removing S110 is likely not deleterious. Second, mutants T114S and T114A are completely inactive, but aromatic substitutes such as T114V and T114I are fully active. These results indicate no HBond is necessary at this location. To reach $S_{\text {cen, }}$ the $S_{\text {int }} F^{-}$has to pass a hydrophobic gate formed by T114, I48, L52, V85', and F88'. T114S may not be bulky enough to preserve gate integrity. We speculate the HBonds with S110 or S84' could help stabilize the dehydrating $\mathrm{F}^{-}$passing the gate $(\triangle \mathrm{ZCOM}$ of $-8 \AA$, Fig. 2B). Although Fluc is insensitive to mutations S84A or S110A $(8,11)$, it would be interesting to test in vitro if the double mutant S84A/S110A is active. Third, H106 is immutable in Fluc-Ec2 as neither aromatic nor polar substitution is tolerated. H106 plays the same role as N41 via an ion-pair with $\mathrm{F}^{-}$(Fig. 3B) in our simulations, which seemingly explains its irreplaceability, but it is unknown why H106 is not strictly conserved. S102 and H106 are respectively present as Y104 and S108 in Fluc-Bpe (Fig. 1D), a pattern shared by FEX Sc $_{\text {(Y345 and S349). It is surprising that FEX }}$ sc tolerates mutations S349A, S349V, and Y345F but is highly sensitive to Y345A (12). In Fluc-Bpe, Y104 only permits the conservative mutation Y104F, but not replacements by Ser, His, or Ile (19). As $\mathrm{F}^{-}$likely coordinates a Tyr side-chain via both anion- $\pi$ pair and hydroxyl HBond, these results imply that if the interactions with residue 106 (Fluc-Ec2 numbering) are reduced, stabilization from residue 102 becomes essential. In other words, the conserved polarity requires precise geometrical side-chain arrangement. Furthermore, two strongly conserved residues, T37 (T39 in Fluc-Bpe) and E86, are found in proximity to H106. In Fluc-Bpe, T39 is replaceable by Ser, but not by Ala, Asn, Cys, or Val, whereas E88 tolerates Ala, Asp, and Gln but not Lys (19). Mutating their FEXsc equivalences causes severe function loss (12). T37 is the sole stable HBond donor around $\mathrm{S}_{\text {out }}\left(\mathbf{F i g}\right.$. 3B). Crystal structures capture $\mathrm{F}^{-}$coordination with E86 (Fig. S3A), but our simulations found no close contact between the two (Fig. S3B) because both were charged. A recent study found that E86 does not ionize between $\mathrm{pH} 7$ and 8.7 and suggests that E86 contributes to anion recognition (19). CpHMD predicted an E86 $\mathrm{p} K_{\mathrm{a}}$ that accords with the experiment ( 4, Table 1). Although the possibility of transient protonation on either E86 or $\mathrm{F}^{-}$by accepting a proton from surrounding water molecules cannot be readily excluded at this stage and may be the focus of future work, it should not negate the major conclusion of the current work; that is, Fluc transfers $\mathrm{F}^{-}$.

Our study also offers atomistic insight into the remarkable selectivity for $\mathrm{F}^{-}$. The $\mathrm{R} 19$ residue generates a positive "Coulombic plug" at either end of the pore and explains the selectivity for $\mathrm{F}^{-}$over $\mathrm{Na}^{+}(6)$. This

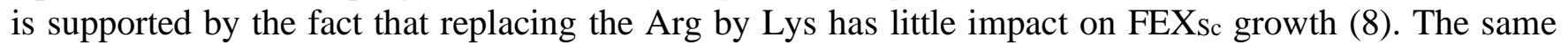
feature is seen in a cationic transporter EmrE which comprises a conserved E14 at a spatially similar location $(85,86)$. Although pore size may also be a factor, the interaction patterns during the transfer (Fig. 
3AB) suggest that $S_{\text {cen }}$ is more likely to be the selectivity filter for $\mathrm{F}^{-}$over $\mathrm{Cl}^{-}(6,8)$. The $\mathrm{F}^{-}$anion is mainly stabilized by HBonds (Fig. 3AB) in this anhydrous (Fig. 2B) region. Considering the weaker HBonding capability of $\mathrm{Cl}^{-}$than that of $\mathrm{F}^{-}(87,88)$, it should be improbable for $\mathrm{Cl}^{-}$to offset the desolvation penalty

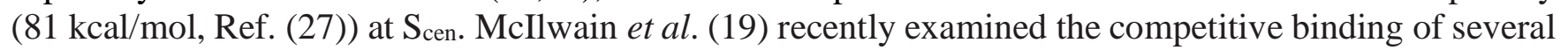
halides and pseudohalides to Sout and discovered that the anion's ability of accepting a HBond contributes to its recognition by Flucs. Our study lends credence to the notion that HBond is the key to the selectivity of Flucs among anions.

Another extraordinary feature of Fluc is the $\mathrm{Na}^{+}$buried at the dimer interface (Fig. 1A) and liganded with four backbone carbonyl oxygen atoms from both monomers (G75s and S78s, Fig. S6A; G77s and T80s in Fluc-Bpe). These $\mathrm{Na}^{+}-\mathrm{O}=\mathrm{C}$ pairs were quite stable in our simulations (Fig. S6B), making $\mathrm{Na}^{+}$a cation that may help keep the two monomers closely "glued". The same observation was reported by ArizExtreme et al. (89). In addition, it was curious that $\mathrm{Na}^{+}$is tetrahedrally coordinated in Fluc crystal structures $(5,14,16)$, as $\mathrm{Na}^{+}$has a typical coordination number $\left(\mathrm{N}_{\text {coord }}\right)$ of $5-6$. We found the $\mathrm{Na}^{+}$was always liganded with one water molecule (Fig. S6C) and occasionally with another water molecule, the carbonyl oxygens of G76s, or the side-chain hydroxyl oxygens of S78s, leading to a stable $\mathrm{N}_{\text {coord }}$ of $5-6$ (Fig. S6D).

Electronically polarizable models can provide a more physically realistic representation for simulating ion transport across heterogeneous membrane systems with complex dielectric environments (29-32). In this study, we have compared the nonpolarizable additive C36m and polarizable Drude-2013 FFs. A significantly overestimated transfer barrier around $S_{\text {cen }}$ (Fig. 2A) and consequently a greatly underestimated flux (Table 2) were given by the additive simulations, consistent with existing computational studies in cation channels (33-36,38-40). A breakdown by residue of the $\mathrm{F}^{-}-$Fluc Einter indicates the additive overstabilization primarily originates from the Coulombic interactions with R19s, which was $10-20 \mathrm{kcal} / \mathrm{mol}$ stronger in C36m (Fig. S2, S7-S9). It is not surprising to see more substantial charge-charge interactions in additive FFs because dielectric constants are underestimated $(31,32)$. However, no large difference was found for the interactions with H106 (Fig. S2, S7-S9). In the case of interactions with $\pi$ electron-rich residues such as F80s, F83s or charged E86s, C36m captured little interaction, but Drude yielded interactions of $5-20 \mathrm{kcal} / \mathrm{mol}$ (Fig. S2) dominantly contributed by Eself $_{\text {s. }}$ (Fig. S3-S4), consistent with previous studies $(89,90)$. This difference is due to a limited description of the ion-induced polarization of $\pi$ electron in additive FFs (61), which Eself accounts for in Drude FFs $(31,79)$. Because F80 ${ }^{\prime}$ HBonds with the $S_{c e n} F^{-}$(Fig. 3AB), Eelec is much stronger around $S_{\text {cen }}($ Fig. S4). But Eself dominates when $\mathrm{F}^{-}$transits between $\mathrm{S}_{\text {cen }}$ and $\mathrm{S}_{\text {ext }}$ (Fig. S4). During the transit, polar residue N41 also experiences an $\mathrm{E}_{\text {self }}$ of up to $10 \mathrm{kcal} / \mathrm{mol}$ (Fig. S9), but $\mathrm{E}_{\mathrm{elec}}$ is essential (Fig. S2, S8) due to the HBond with $\mathrm{F}^{-}$(Fig. 3AB).

In summary, we have computationally investigated the fluoride transport mechanism through Fluc-Ec 2. We confirm $\mathrm{F}^{-}$transfer with a calculated rate in encouraging agreement with experiment and we reveal that the transfer is enabled by $\mathrm{F}^{-}$relaying inside a non-bonded network. Although the variance in the porelining polar residues remains somewhat puzzling, the present work paves the way for future studies to elucidate delicate control of the pore polarity for rapid $\mathrm{F}^{-}$transit. Lastly, this work highlights the possible importance of electronic polarization during anion permeation through membrane channels/transporters and the necessity of using polarizable models for treating such processes.

\section{SUPPORTING MATERIAL}

Supporting material can be found online at https://doi.org/10.1016/j.bpj.xxxx.xx.xxx. 


\section{AUTHOR CONTRIBUTIONS}

G.A.V. designed the research. Z.W. and Z.Y. ran the simulations. Z.W., Z.Y., and G.A.V. analyzed the data. Z.W., Z.Y., and G.A.V. wrote the article.

\section{ACKNOWLEDGMENTS}

This research was supported by the National Institute of General Medical Sciences (NIGMS) of the National Institutes of Health (NIH Grant R01 GM053148). The computational resources were provided by the University of Chicago Research Computing Center (RCC).

\section{SUPPORTING CITATIONS}

References (91-113) appear in the Supporting material.

\section{REFERENCES}

1. Weinstein, L. H., and A. Davison. 2004. Fluorides in the Environment: Effects on Plants and Animals, 1 st ed. CABI Publishing, Cambridge, MA.

2. Marquis, R. E. 1995. Antimicrobial actions of fluoride for oral bacteria. Can. J. Microbiol. 41:955-964.

3. Marquis, R. E., S. A. Clock, and M. Mota-Meira. 2003. Fluoride and organic weak acids as modulators of microbial physiology. FEMS Microbiol. Rev. 26:493-510.

4. Stockbridge, R. B., H.-H. Lim, R. Otten, C. Williams, T. Shane, Z. Weinberg, and C. Miller. 2012. Fluoride resistance and transport by riboswitch-controlled CLC antiporters. Proc. Natl. Acad. Sci. U.S.A. 109:1528915294.

5. Macdonald, C. B., and R. B. Stockbridge. 2017. A topologically diverse family of fluoride channels. Curr. Opin. Struct. Biol. 45:142-149.

6. Stockbridge, R. B., J. L. Robertson, L. Kolmakova-Partensky, and C. Miller. 2013. A family of fluoridespecific ion channels with dual-topology architecture. eLife 2:e01084.

7. Ji, C. H., R. B. Stockbridge, and C. Miller. 2014. Bacterial fluoride resistance, Fluc channels, and the weak acid accumulation effect. J. Gen. Physiol. 144:257-261.

8. Smith, K. D., P. B. Gordon, A. Rivetta, K. E. Allen, T. Berbasova, C. Slayman, and S. A. Strobel. 2015. Yeast Fex1p Is a Constitutively Expressed Fluoride Channel with Functional Asymmetry of Its Two Homologous Domains. J. Biol. Chem. 290:19874-19887.

9. Turman, D. L., J. T. Nathanson, R. B. Stockbridge, T. O. Street, and C. Miller. 2015. Two-sided block of a dual-topology $\mathrm{F}^{-}$channel. Proc. Natl. Acad. Sci. U.S.A. 112:5697-5701.

10. Last, N. B., L. Kolmakova-Partensky, T. Shane, and C. Miller. 2016. Mechanistic signs of double-barreled structure in a fluoride ion channel. eLife 5:e18767.

11. Last, N. B., S. Sun, M. C. Pham, and C. Miller. 2017. Molecular determinants of permeation in a fluoridespecific ion channel. eLife 6:e31259.

12. Berbasova, T., S. Nallur, T. Sells, K. D. Smith, P. B. Gordon, S. L. Tausta, and S. A. Strobel. 2017. Fluoride export (FEX) proteins from fungi, plants and animals are 'single barreled' channels containing one functional and one vestigial ion pore. PLOS ONE 12:e0177096.

13. Turman, D. L., and R. B. Stockbridge. 2017. Mechanism of single- and double-sided inhibition of dual topology fluoride channels by synthetic monobodies. J. Gen. Physiol. 149:511-522.

14. McIlwain, B. C., K. Martin, E. A. Hayter, and R. B. Stockbridge. 2020. An Interfacial Sodium Ion is an Essential Structural Feature of Fluc Family Fluoride Channels. J. Mol. Biol. 432:1098-1108. 
15. Stockbridge, R. B., A. Koide, C. Miller, and S. Koide. 2014. Proof of dual-topology architecture of Fluc Fchannels with monobody blockers. Nat. Commun. 5:5120.

16. Stockbridge, R. B., L. Kolmakova-Partensky, T. Shane, A. Koide, S. Koide, C. Miller, and S. Newstead. 2015. Crystal structures of a double-barrelled fluoride ion channel. Nature 525:548-551.

17. Turman, D. L., A. Z. Cheloff, A. D. Corrado, J. T. Nathanson, and C. Miller. 2018. Molecular Interactions between a Fluoride Ion Channel and Synthetic Protein Blockers. Biochemistry 57:1212-1218.

18. McIlwain, B. C., S. Newstead, and R. B. Stockbridge. 2018. Cork-in-Bottle Occlusion of Fluoride Ion Channels by Crystallization Chaperones. Structure 26:635-639.

19. McIlwain, B. C., R. Gundepudi, B. B. Koff, and R. B. Stockbridge. 2021. The fluoride permeation pathway and anion recognition in Fluc family fluoride channels. eLife 10:e69482.

20. Sand, O., M. Gingras, N. Beck, C. Hall, and N. Trun. 2003. Phenotypic characterization of overexpression or deletion of the Escherichia coli crcA, cspE and $\operatorname{crcB}$ genes. Microbiology 149:2107-2117.

21. Rapp, M., E. Granseth, S. Seppälä, and G. von Heijne. 2006. Identification and evolution of dual-topology membrane proteins. Nat. Struct. Mol. Biol. 13:112-116.

22. Weinberg, Z., J. X. Wang, J. Bogue, J. Yang, K. Corbino, R. H. Moy, and R. R. Breaker. 2010. Comparative genomics reveals 104 candidate structured RNAs from bacteria, archaea, and their metagenomes. Genome Biol. 11:R31.

23. Baker, J. L., N. Sudarsan, Z. Weinberg, A. Roth, R. B. Stockbridge, and R. R. Breaker. 2012. Widespread Genetic Switches and Toxicity Resistance Proteins for Fluoride. Science 335:233-235.

24. Li, S., K. D. Smith, J. H. Davis, P. B. Gordon, R. R. Breaker, and S. A. Strobel. 2013. Eukaryotic resistance to fluoride toxicity mediated by a widespread family of fluoride export proteins. Proc. Natl. Acad. Sci. U.S.A. 110:19018-19023.

25. Smart, O. S., J. G. Neduvelil, X. Wang, B. A. Wallace, and M. S. P. Sansom. 1996. HOLE: A program for the analysis of the pore dimensions of ion channel structural models. J. Mol. Graph. 14:354-360.

26. Lomize, M. A., I. D. Pogozheva, H. Joo, H. I. Mosberg, and A. L. Lomize. 2012. OPM database and PPM web server: resources for positioning of proteins in membranes. Nucleic Acids Res. 40:D370-D376.

27. Marcus, Y. 1991. Thermodynamics of Solvation of Ions. Part 5.- Gibbs Free Energy of Hydration at 298.15 K. J. Chem. Soc. Faraday Trans. 87:2995-2999.

28. Song, J. L., C. C. Hou, J. X. Guo, Q. Niu, X. H. Wang, Z. J. Ren, Q. Zhang, C. X. Feng, L. Y. Liu, W. Tian, and L. G. Li. 2020. Two New Members of CsFEXs Couple Proton Gradients to Export Fluoride and Participate in Reducing Fluoride Accumulation in Low-Fluoride Tea Cultivars. J. Agric. Food Chem. 68:8568-8579.

29. Flood, E., C. Boiteux, B. Lev, I. V. Vorobyov, and T. W. Allen. 2019. Atomistic Simulations of Membrane Ion Channel Conduction, Gating, and Modulation. Chem. Rev. 119:7737-7832.

30. Maffeo, C., S. Bhattacharya, J. Yoo, D. Wells, and A. Aksimentiev. 2012. Modeling and Simulation of Ion Channels. Chem. Rev. 112:6250-6284.

31. Lemkul, J. A., J. Huang, B. Roux, and A. D. MacKerell Jr. 2016. An Empirical Polarizable Force Field Based on the Classical Drude Oscillator Model: Development History and Recent Applications. Chem. Rev. 116:4983-5013.

32. Jing, Z., C. Liu, S. Y. Cheng, R. Qi, B. D. Walker, J.-P. Piquemal, and P. Ren. 2019. Polarizable Force Fields for Biomolecular Simulations: Recent Advances and Applications. Annu. Rev. Biophys. 48:371-394.

33. Patel, S., J. E. Davis, and B. A. Bauer. 2009. Exploring Ion Permeation Energetics in Gramicidin A Using Polarizable Charge Equilibration Force Fields. J. Am. Chem. Soc. 131:13890-13891.

34. Vorobyov, I., B. Bekker, and T. W. Allen. 2010. Electrostatics of Deformable Lipid Membranes. Biophys. J. 98:2904-2913.

35. Peng, X. D., Y. B. Zhang, H. Y. Chu, Y. Li, D. L. Zhang, L. R. Cao, and G. H. Li. 2016. Accurate Evaluation of Ion Conductivity of the Gramicidin A Channel Using a Polarizable Force Field without Any Corrections. $J$. Chem. Theory Comput. 12:2973-2982.

36. Manin, N., M. C. da Silva, I. Zdravkovic, O. Eliseeva, A. Dyshin, O. Yaşar, D. R. Salahub, A. M. Kolker, M. G. Kiselev, and S. Y. Noskov. 2016. LiCl solvation in $N$-methyl-acetamide (NMA) as a model for understanding $\mathrm{Li}^{+}$binding to an amide plane. Phys. Chem. Chem. Phys. 18:4191-4200. 
37. Zhang, Y., K. Haider, D. Kaur, V. A. Ngo, X. Cai, J. Mao, U. Khaniya, X. Zhu, S. Noskov, T. Lazaridis, and M. R. Gunner. 2021. Characterizing the Water Wire in the Gramicidin Channel Found by Monte Carlo Sampling Using Continuum Electrostatics and in Molecular Dynamics Trajectories with Conventional or Polarizable Force Fields. J. Comput. Biophys. Chem. 20:111-130.

38. Ngo, V., H. Li, A. D. MacKerell Jr, T. W. Allen, B. Roux, and S. Noskov. 2021. Polarization Effects in WaterMediated Selective Cation Transport across a Narrow Transmembrane Channel. J. Chem. Theory Comput. 17:1726-1741.

39. Jing, Z., J. A. Rackers, L. R. Pratt, C. Liu, S. B. Rempe, and P. Ren. 2021. Thermodynamics of ion binding and occupancy in potassium channels. Chem. Sci. 12:8920-8930.

40. Sun, R.-N., and H. Gong. 2017. Simulating the Activation of Voltage Sensing Domain for a Voltage-Gated Sodium Channel Using Polarizable Force Field. J. Phys. Chem. Lett. 8:901-908.

41. Vergara-Jaque, A., P. Fong, and J. Comer. 2017. Iodide Binding in Sodium-Coupled Cotransporters. J. Chem. Inf. Model. 57:3043-3055.

42. Lee, M. S., F. R. Salsbury Jr., and C. L. Brooks III. 2004. Constant-pH Molecular Dynamics Using Continuous Titration Coordinates. Proteins 56:738-752.

43. Khandogin, J., and C. L. Brooks III. 2005. Constant pH Molecular Dynamics with Proton Tautomerism. Biophys. J. 89:141-157.

44. Wallace, J. A., and J. K. Shen. 2011. Continuous Constant pH Molecular Dynamics in Explicit Solvent with pH-Based Replica Exchange. J. Chem. Theory Comput. 7:2617-2629.

45. Chen, W., Y. Huang, and J. Shen. 2016. Conformational Activation of a Transmembrane Proton Channel from Constant pH Molecular Dynamics. J. Phys. Chem. Lett. 7:3961-3966.

46. Chen, W., B. H. Morrow, C. Y. Shi, and J. K. Shen. 2014. Recent development and application of constant $\mathrm{pH}$ molecular dynamics. Mol. Simul. 40:830-838.

47. Torrie, G. M., and J. P. Valleau. 1977. Nonphysical Sampling Distributions in Monte Carlo Free-Energy Estimation: Umbrella Sampling. J. Comput. Phys. 23:187-199.

48. Sugita, Y., A. Kitao, and Y. Okamoto. 2000. Multidimensional replica-exchange method for free-energy calculations. J. Chem. Phys. 113:6042-6051.

49. Zhang, C., D. Bell, M. Harger, and P. Ren. 2017. Polarizable Multipole-Based Force Field for Aromatic Molecules and Nucleobases. J. Chem. Theory Comput. 13:666-678.

50. Rupakheti, C. R., B. Roux, F. Dehez, and C. Chipot. 2018. Modeling induction phenomena in amino acid cation $-\pi$ interactions. Theor. Chem. Acc. 137:174.

51. Inakollu, V. S. S., D. P. Geerke, C. N. Rowley, and H. Yu. 2020. Polarisable force fields: what do they add in biomolecular simulations? Curr. Opin. Struct. Biol. 61:182-190.

52. Klauda, J. B., R. M. Venable, J. A. Freites, J. W. O’Connor, D. J. Tobias, C. Mondragon-Ramirez, I. V. Vorobyov, A. D. MacKerell Jr., and R. W. Pastor. 2010. Update of the CHARMM All-Atom Additive Force Field for Lipids: Validation on Six Lipid Types. J. Phys. Chem. B 114:7830-7843.

53. Best, R. B., X. Zhu, J. Shim, P. E. M. Lopes, J. Mittal, M. Feig, and A. D. MacKerell Jr. 2012. Optimization of the Additive CHARMM All-Atom Protein Force Field Targeting Improved Sampling of the Backbone $\varphi, \psi$ and Side-Chain $\chi_{1}$ and $\chi_{2}$ Dihedral Angles. J. Chem. Theory Comput. 8:3257-3273.

54. Huang, J., S. Rauscher, G. Nawrocki, T. Ran, M. Feig, B. L. de Groot, H. Grubmüller, and A. D. MacKerell Jr. 2017. CHARMM36m: an improved force field for folded and intrinsically disordered proteins. Nat. Methods 14:71-73.

55. Lamoureux, G., E. Harder, I. V. Vorobyov, B. Roux, and A. D. MacKerell Jr. 2006. A polarizable model of water for molecular dynamics simulations of biomolecules. Chem. Phys. Lett. 418:245-249.

56. Lamoureux, G., and B. Roux. 2006. Absolute Hydration Free Energy Scale for Alkali and Halide Ions Established from Simulations with a Polarizable Force Field. J. Phys. Chem. B 110:3308-3322.

57. Yu, H., T. W. Whitfield, E. Harder, G. Lamoureux, I. V. Vorobyov, V. M. Anisimov, A. D. MacKerell Jr., and B. Roux. 2010. Simulating Monovalent and Divalent Ions in Aqueous Solution Using a Drude Polarizable Force Field. J. Chem. Theory Comput. 6:774-786. 
58. Chowdhary, J., E. Harder, P. E. M. Lopes, L. Huang, A. D. MacKerell Jr., and B. Roux. 2013. A Polarizable Force Field of Dipalmitoylphosphatidylcholine Based on the Classical Drude Model for Molecular Dynamics Simulations of Lipids. J. Phys. Chem. B 117:9142-9160.

59. Lopes, P. E. M., J. Huang, J. Shim, Y. Luo, H. Li, B. Roux, and A. D. MacKerell Jr. 2013. Polarizable Force Field for Peptides and Proteins Based on the Classical Drude Oscillator. J. Chem. Theory Comput. 9:54305449.

60. Li, H., J. Chowdhary, L. Huang, X. He, A. D. MacKerell Jr., and B. Roux. 2017. Drude Polarizable Force Field for Molecular Dynamics Simulations of Saturated and Unsaturated Zwitterionic Lipids. J. Chem. Theory Comput. 13:4535-4552.

61. Lin, F.-Y., and A. D. MacKerell Jr. 2020. Improved Modeling of Cation- $\pi$ and Anion-Ring Interactions Using the Drude Polarizable Empirical Force Field for Proteins. J. Comput. Chem. 41:439-448.

62. Yue, Z., W. Chen, H. I. Zgurskaya, and J. Shen. 2017. Constant pH Molecular Dynamics Reveals How Proton Release Drives the Conformational Transition of a Transmembrane Efflux Pump. J. Chem. Theory Comput. 13:6405-6414.

63. Yue, Z., C. Li, G. A. Voth, and J. M. J. Swanson. 2019. Dynamic Protonation Dramatically Affects the Membrane Permeability of Drug-like Molecules. J. Am. Chem. Soc. 141:13421-13433.

64. Durell, S. R., B. R. Brooks, and A. Ben-Naim. 1994. Solvent-Induced Forces between Two Hydrophilic Groups. J. Phys. Chem. 98:2198-2202.

65. Jorgensen, W. L., J. Chandrasekhar, J. D. Madura, R. W. Impey, and M. L. Klein. 1983. Comparison of simple potential functions for simulating liquid water. J. Chem. Phys. 79:926-935.

66. Senn, H. M., D. O'Hagan, and W. Thiel. 2005. Insight into Enzymatic C-F Bond Formation from QM and QM/MM Calculations. J. Am. Chem. Soc. 127:13643-13655.

67. Laage, D., H. Demirdjian, and J. T. Hynes. 2005. Intermolecular vibration-vibration energy transfer in solution: Hydrogen fluoride in water. Chem. Phys. Lett. 405:453-458.

68. MacKerell Jr., A. D., D. Bashford, M. Bellott, R. L. Dunbrack Jr., J. D. Evanseck, M. J. Field, S. Fischer, J. Gao, H. Guo, S. Ha, D. Joseph-McCarthy, L. Kuchnir, K. Kuczera, F. T. Lau, C. Mattos, S. Michnick, T. Ngo, D. T. Nguyen, B. Prodhom, W. E. Reiher, B. Roux, M. Schlenkrich, J. C. Smith, R. Stote, J. Straub, M. Watanabe, J. Wiórkiewicz-Kuczera, D. Yin, and M. Karplus. 1998. All-Atom Empirical Potential for Molecular Modeling and Dynamics Studies of Proteins. J. Phys. Chem. B 102:3586-3616.

69. Mackerell Jr., A. D., M. Feig, and C. L. Brooks III. 2004. Extending the Treatment of Backbone Energetics in Protein Force Fields: Limitations of Gas-Phase Quantum Mechanics in Reproducing Protein Conformational Distributions in Molecular Dynamics Simulations. J. Comput. Chem. 25:1400-1415.

70. Jo, S., T. Kim, and W. Im. 2007. Automated Builder and Database of Protein/Membrane Complexes for Molecular Dynamics Simulations. PLOS ONE 2:e880.

71. Jo, S., T. Kim, V. G. Iyer, and W. Im. 2008. CHARMM-GUI: A Web-Based Graphical User Interface for CHARMM. J. Comput. Chem. 29:1859-1865.

72. Jo, S., J. B. Lim, J. B. Klauda, and W. Im. 2009. CHARMM-GUI Membrane Builder for Mixed Bilayers and Its Application to Yeast Membranes. Biophys. J. 97:50-58.

73. Wu, E. L., X. Cheng, S. Jo, H. Rui, K. C. Song, E. M. Dávila-Contreras, Y. Qi, J. Lee, V. Monje-Galvan, R. M. Venable, J. B. Klauda, and W. Im. 2014. CHARMM-GUI Membrane Builder Toward Realistic Biological Membrane Simulations. J. Comput. Chem. 35:1997-2004.

74. Lee, J., X. Cheng, J. M. Swails, M. S. Yeom, P. K. Eastman, J. A. Lemkul, S. Wei, J. Buckner, J. C. Jeong, Y. Qi, S. Jo, V. S. Pande, D. A. Case, C. L. Brooks III, A. D. MacKerell Jr., J. B. Klauda, and W. Im. 2016. CHARMM-GUI Input Generator for NAMD, GROMACS, AMBER, OpenMM, and CHARMM/OpenMM Simulations Using the CHARMM36 Additive Force Field. J. Chem. Theory Comput. 12:405-413.

75. Abraham, M. J., T. Murtola, R. Schulz, S. Páll, J. C. Smith, B. Hess, and E. Lindahl. 2015. GROMACS: High performance molecular simulations through multi-level parallelism from laptops to supercomputers. SoftwareX $1-2: 19-25$.

76. Brooks, B. R., C. L. Brooks III, A. D. Mackerell, L. Nilsson, R. J. Petrella, B. Roux, Y. Won, G. Archontis, C. Bartels, S. Boresch, A. Caflisch, L. Caves, Q. Cui, A. R. Dinner, M. Feig, S. Fischer, J. Gao, M. Hodoscek, 
W. Im, K. Kuczera, T. Lazaridis, J. Ma, V. Ovchinnikov, E. Paci, R. W. Pastor, C. B. Post, J. Z. Pu, M. Schaefer, B. Tidor, R. M. Venable, H. L. Woodcock, X. Wu, W. Yang, D. M. York, and M. Karplus. 2009. CHARMM: The Biomolecular Simulation Program. J. Comput. Chem. 30:1545-1614.

77. Phillips, J. C., R. Braun, W. Wang, J. Gumbart, E. Tajkhorshid, E. Villa, C. Chipot, R. D. Skeel, L. Kalé, and K. Schulten. 2005. Scalable Molecular Dynamics with NAMD. J. Comput. Chem. 26:1781-1802.

78. Thurlkill, R. L., G. R. Grimsley, J. M. Scholtz, and C. N. Pace. 2006. pK values of the ionizable groups of proteins. Protein Sci. 15:1214-1218.

79. Lin, F.-Y., and A. D. MacKerell Jr. 2019. Improved Modeling of Halogenated Ligand-Protein Interactions Using the Drude Polarizable and CHARMM Additive Empirical Force Fields. J. Chem. Inf. Model. 59:215228.

80. Woolf, T. B., and B. Roux. 1994. Conformational Flexibility of $o$-Phosphorylcholine and $o$ Phosphorylethanolamine: A Molecular Dynamics Study of Solvation Effects. J. Am. Chem. Soc. 116:59165926.

81. Vanýsek, P. 2006. Ionic Conductivity and Diffusion at Infinite Dilution. In CRC Handbook of Chemistry and Physics, 87th ed.; R. L. David, Ed. CRC Press, Boca Raton, FL; pp. 76-78.

82. Wang, J. H. 1954. Effect of Ions on the Self-Diffusion and Structure of Water in Aqueous Electrolytic Solutions. J. Phys. Chem. 58:686-692.

83. Prajapati, J. D., C. Mele, M. A. Aksoyoglu, M. Winterhalter, and U. Kleinekathöfer. 2020. Computational Modeling of Ion Transport in Bulk and through a Nanopore Using the Drude Polarizable Force Field. J. Chem. Inf. Model. 60:3188-3203.

84. Szabo, A., K. Schulten, and Z. Schulten. 1980. First passage time approach to diffusion controlled reactions. J. Chem. Phys. 72:4350-4357.

85. Muth, T. R., and S. Schuldiner. 2000. A membrane-embedded glutamate is required for ligand binding to the multidrug transporter EmrE. EMBO J. 19:234-240.

86. Yerushalmi, H., and S. Schuldiner. 2000. An Essential Glutamyl Residue in EmrE, a Multidrug Antiporter from Escherichia coli. J. Biol. Chem. 275:5264-5269.

87. Emsley, J. 1980. Very Strong Hydrogen Bonding. Chem. Soc. Rev. 9:91-124.

88. Chowdhuri, S., and A. Chandra. 2006. Dynamics of Halide Ion-Water Hydrogen Bonds in Aqueous Solutions: Dependence on Ion Size and Temperature. J. Phys. Chem. B 110:9674-9680.

89. Ariz-Extreme, I., and J. S. Hub. 2018. Assigning crystallographic electron densities with free energy calculations-The case of the fluoride channel Fluc. PLoS ONE 13:e0196751.

90. Jackson, M. R., R. Beahm, S. Duvvuru, C. Narasimhan, J. Wu, H. N. Wang, V. M. Philip, R. J. Hinde, and E. E. Howell. 2007. A Preference for Edgewise Interactions between Aromatic Rings and Carboxylate Anions: The Biological Relevance of Anion-Quadrupole Interactions. J. Phys. Chem. B 111:8242-8249.

91. Nosé, S. 1984. A molecular dynamics method for simulations in the canonical ensemble. Mol. Phys. 52:255268.

92. Hoover, W. G. 1985. Canonical dynamics: Equilibrium phase-space distributions. Phys. Rev. A 31:1695-1697.

93. Parrinello, M., and A. Rahman. 1981. Polymorphic transitions in single crystals: A new molecular dynamics method. J. Appl. Phys. 52:7182-7190.

94. Olsson, M. H. M., C. R. Søndergaard, M. Rostkowski, and J. H. Jensen. 2011. PROPKA3: Consistent Treatment of Internal and Surface Residues in Empirical $\mathrm{p} K_{\mathrm{a}}$ Predictions. J. Chem. Theory Comput. 7:525537.

95. Søndergaard, C. R., M. H. M. Olsson, M. Rostkowski, and J. H. Jensen. 2011. Improved Treatment of Ligands and Coupling Effects in Empirical Calculation and Rationalization of $\mathrm{p} K_{\mathrm{a}}$ Values. J. Chem. Theory Comput. 7:2284-2295.

96. Hess, B., H. Bekker, H. J. C. Berendsen, and J. G. E. M. Fraaije. 1997. LINCS: A Linear Constraint Solver for Molecular Simulations. J. Comput. Chem. 18:1463-1472.

97. Darden, T., D. York, and L. Pedersen. 1993. Particle mesh Ewald: An $N \bullet \log (N)$ method for Ewald sums in large systems. J. Chem. Phys. 98:10089-10092. 
98. Essmann, U., L. Perera, M. L. Berkowitz, T. Darden, H. Lee, and L. G. Pedersen. 1995. A smooth particle mesh Ewald method. J. Chem. Phys. 103:8577-8593.

99. Wang, Z., J. M. J. Swanson, and G. A. Voth. 2018. Modulating the Chemical Transport Properties of a Transmembrane Antiporter via Alternative Anion Flux. J. Am. Chem. Soc. 140:16535-16543.

100. Wang, Z., J. M. J. Swanson, and G. A. Voth. 2020. Local Conformational Dynamics Regulating Transport Properties of a $\mathrm{Cl}^{-} / \mathrm{H}^{+}$Antiporter. J. Comput. Chem. 41:513-519.

101. Ryckaert, J.-P., G. Ciccotti, and H. J. C. Berendsen. 1977. Numerical Integration of the Cartesian Equations of Motion of a System with Constraints: Molecular Dynamics of n-Alkanes. J. Comput. Phys. 23:327-341.

102. Feller, S. E., Y. Zhang, R. W. Pastor, and B. R. Brooks. 1995. Constant pressure molecular dynamics simulation: The Langevin piston method. J. Chem. Phys. 103:4613-4621.

103. Im, W., M. S. Lee, and C. L. Brooks III. 2003. Generalized Born Model with a Simple Smoothing Function. J. Comput. Chem. 24:1691-1702.

104. Im, W., M. Feig, and C. L. Brooks III. 2003. An Implicit Membrane Generalized Born Theory for the Study of Structure, Stability, and Interactions of Membrane Proteins. Biophys. J. 85:2900-2918.

105. Chen, J., W. Im, and C. L. Brooks III. 2006. Balancing Solvation and Intramolecular Interactions: Toward a Consistent Generalized Born Force Field. J. Am. Chem. Soc. 128:3728-3736.

106. Miyamoto, S., and P. A. Kollman. 1992. SETTLE: An Analytical Version of the SHAKE and RATTLE Algorithm for Rigid Water Models. J. Comput. Chem. 13:952-962.

107. Liang, R., H. Li, J. M. J. Swanson, and G. A. Voth. 2014. Multiscale simulation reveals a multifaceted mechanism of proton permeation through the influenza A M2 proton channel. Proc. Natl. Acad. Sci. U.S.A. 111:9396-9401.

108. Hill, A. V. 1910. The possible effects of the aggregation of the molecules of haemoglobin on its dissociation curves. J. Physiol. 40:iv-vii.

109. Kumar, S., J. M. Rosenberg, D. Bouzida, R. H. Swendsen, and P. A. Kollman. 1992. The Weighted Histogram Analysis Method for Free-Energy Calculations on Biomolecules. I. The Method. J. Comput. Chem. 13:10111021.

110. De Loof, H., L. Nilsson, and R. Rigler. 1992. Molecular Dynamics Simulation of Galanin in Aqueous and Nonaqueous Solution. J. Am. Chem. Soc. 114:4028-4035.

111. Kumar, S., and R. Nussinov. 2002. Close-Range Electrostatic Interactions in Proteins. ChemBioChem 3:604617.

112. Chakravarty, S., A. R. Ung, B. Moore, J. Shore, and M. Alshamrani. 2018. A Comprehensive Analysis of Anion-Quadrupole Interactions in Protein Structures. Biochemistry 57:1852-1867.

113. Humphrey, W., A. Dalke, and K. Schulten. 1996. VMD: Visual Molecular Dynamics. J. Mol. Graph. 14:3338. 